\section{Two cases of aspergillus endo- carditis in non neutropenic children on chemotherapy for acute lymphoblastic leukaemia}

\author{
Emmanouil Nikolousis, Mark Velangi \\ Haematology department, Birmingham \\ Childrens Hospital, United Kingdom
}

\section{Abstract}

Fungal endocarditis (FE) is a rare complication in immunocompromised patients which is difficult to diagnose and has been characterized by excessive mortality ( $>50 \%$ ) and morbidity, regardless of treatment. The lack of clinical trials due to the small number of cases contributes further to a poor outcome. In our two cases of aspergilllus endocarditis we reviewed the clinical features, echocardiographic findings, microbiologic data, treatment, and outcome of these 2 cases and provide a current characterization of the syndrome. In this paper we have demonstrated the diversity of presentation of a critical fungal infection in immunocompromised but non neutropenic paediatric patients. The prompt diagnosis and initiation of treatment is crucial for a favourable outcome along with the use of double antifungal treatment with liposomal amphotericin and voriconazole initially which could be later switched to oral voriconazole with a good tissue penetration. Histological samples as well as radiological evidence and echocardiograms should be reviewed by experienced clinicians in order to aid diagnosis and promptly initiate treatment for these patients in order to achieve a favourable outcome

\section{Introduction}

Fungal endocarditis (FE) is an uncommon occurrence. Previously published series reported fungi as causes of infective endocarditis in $1.3-6 \%$ of cases. FE has been characterized by excessive mortality $(>50 \%)$ and morbidity, regardless of treatment. A combined medicalsurgical approach seems to offer an improved outcome. However, there are no clinical trials to support or refute this opinion, largely because of the rarity of the syndrome Advances in medical and surgical therapies, including reconstructive cardiovascular surgery, implantation of intracardiac prosthetic devices, prolonged use of IV catheters, exposure to broadspectrum antibiotics, and immunosuppression, have been implicated as causes of the increase in the number of cases of fungemia and FE seen. Establishing a definitive diagnosis of infective endocarditis is frequently a problem. ${ }^{1,2}$ Aspergilli are widespread molds populating virtually every site of organic debris. More than 200 different species of Aspergillus are known, but only a few are consistently pathogenic. A. fumigatus and A. flavus are the most frequently isolated species. $^{3}$

\section{Case \#1}

A 2 years old girl was diagnosed with pre BALL with a total white cell count of $43.000 / \mathrm{dL}$ and started treatment on the UKALL2003 trial. During intensification block where patients receive Vincristine along with anthracyclines she was admitted with ongoing pyrexia, tachycardia, rigors and a blanching rash on the left thigh. The full blood count showed a WBC:3.000/dL N:1.800/dL and a high C-reactive protein. As there was no obvious focus of infection and had a Hickman line she was started on broad spectrum antibiotics for presumed line infection according to the regional protocol (meropenem and vancomycin) for immunocompromised patients which was switched to second line antibiotics 48 hours later due to persistent pyrexia. Simultaneously blood cultures were positive for aspergillus fumigatus .Clinical examination revealed a systolic murmur and bilateral crackles. The echocardiogram demonstrated a right atrium/Superior Vena Cava echogenic lesion representing a vegetation. The biopsy of the lesion confirmed the presence of aspergillus fumigatus. The patient underwent a high resolution chest CT scan which didn't reveal any evidence of fungal infection. Hickman line was removed and a temporary neck line was inserted for antifungal treatment. Chemotherapy was withheld. She was commenced on liposomal Amphotericin $3 \mathrm{mg} / \mathrm{kg}$ along with intravenous voriconazole which was switched to oral voriconazole once adequate levels in the blood were reached. Liposomal amphotericin was discontinued after 2 weeks and she remained on oral Voriconazole throughout chemotherapy. An Echocardiogram was repeated after 4 weeks and showed no evidence of vegetations with negative blood cultures. Currently the patient is well and in remission from ALL

\section{Case \#2}

An 11 years old boy diagnosed with Acute Lymphoblastic leukaemia in March 2004. He was treated on UKALL 2003 trial. During his maintenance phase he was admitted to hospi-
Correspondence: Emmanouil Nikolousis, Department,Heart of England NHS Trust,Bordesley Green, Birmingham B9 5SS United Kingdom. Tel. +447702812799.

E-mail: manos.nikolousis@heartofengland.nhs.uk

Key words: Aspergillus endocarditis, leukaemia liposomal amphotericin, voriconazole.

Acknowledgments: authors acknowledge the help of the nursing staff at Birmingham Childrens Hospital.

Conflict of interest: the authors report no conflicts of interest.

Received for publication: 12 April 2011.

Revision received: 8 June 2011.

Accepted for publication: 8 June 2011.

This work is licensed under a Creative Commons Attribution NonCommercial 3.0 License (CC BYNC 3.0).

(C) Copyright E. Nikolousis and M. Velangi, 2011 Licensee PAGEPress, Italy

Hematology Reports 2011; 3:e7

doi:10.4081/hr.2011.e7

tal with pyrexia and shortness of breath suggestive of pneumonia. The full blood count showed WBC:12000/dL N:8.400/dL and a high $\mathrm{C}$-reactive protein. Chest X-ray was normal. He was started on intravenous meropenem as guided by the regional protocol for immunocompromised patients and four days later his temperature was settled as well as his symptoms and in the absence of positive blood cultures he was discharged from hospital on oral clarithromycin. However 5 days later the patient was readmitted with pyrexia, productive cough and the chest X-ray showed right middle lobe consolidation. He was started on intravenous cefuroxime and Azithromycin with minimal improvement. Sputum samples were sent for microscopy and cultures. Two days later he developed a systolic murmur and the antibiotics were changed to benzylpenicillin and gentamycin for presumed endocarditis despite the lack of positive blood cultures. In addition to this Aspergillus fumigatus was grown from the sputum cultures. He had a high resolution CT scan of the chest which showed a cavitation on the right middle lobe representing an aspergilloma. Treatment with intravenous Ambisome was initiated along with intravenous voriconazole which was switched to the oral preparation 5 days later. Unfortunately the patient developed profound symptoms of cardiac failure and thus he had an Echocardiogram which revealed a right ventricular outflow tract (RVOT) vegetation of $2.5 \times 2.0 \mathrm{~cm}$. Due to the patient's marked cardiac compromise the RVOT vegetation was surgically resected and on the histopathologi- 
cal sections there were numerous PAS positive hyphae representing Aspergillus. Cultures confirmed the presence of Aspergillus fumigatus. Post operatively he became hypotensive and required inotropic support in the paediatric intensive care unit. Liposomal Amphotericin was discontinued after 17 days and he was continued on oral voriconazole for three months. He had a follow up high resolution CT scan and an ECHO which confirmed complete resolution of the aspergillomas.

\section{Discussion}

Systemic aspergillus endocarditis occurs mainly in severely immunocompromised patients and in most cases in the literature includes a pulmonary focus and the survival was frequently poor. ${ }^{4}$ Review of the British literature showed five patients with fungal endocarditis in the setting of bone marrow transplantation and revealed the same difficulties in diagnosis and poor outcome. ${ }^{5,6}$ Amphotericin B, as exemplified in the current literature review, remains the mainstay of medical therapy. Mortality caused by Aspergillus endocarditis in recent literature reviews is around $80 \%$, and surgical intervention with valve replacement did not improve mortality rates as compared with rates for patients who received antifungal monotherapy with Amphotericin B. ${ }^{7,8}$ In our first case liposomal Amphotericin B along with voriconazole resolved the aspergillus endocarditis in a month without the need for surgical intervention whereas on the second case due to the cardiocirculatory compromise, urgent resection of the aspergilloma along with combined antifungal therapy was indicated and despite the need for intensive care treatment the outcome was good. In the literature a patient with prosthetic valve endocarditis due to Aspergillus fumigatus was successfully treated with aggressive surgical debridement alone. ${ }^{9}$

The rarity of the syndrome coupled with negative blood culture results in most mold cases and some yeast cases compromises our ability to secure an early diagnosis. ${ }^{10}$ However in our cases Aspergillus fumigatus was isolated from the blood cultures in our first case which was found very rarely in the international literature and from the sputum cultures in our second case enabling us to initiate treatment promptly. Furthermore the large vegetations that characterize this illness seem to increase the sensitivity of transthoracic echocardiogram and should prompt a consideration of fungal endocarditis in the setting of blood culture-negative endocarditis in the immunocompromised patient. ${ }^{11}$

The lack of pulmonary involvement in our first case in the presence of generalized infection with Aspergillus fumigatus is unique, since most of the cases reported to date included severe pulmonary disease. Despite the lack of clinical and pathological signs of a pulmonary port of entry, infection through the respiratory tract cannot be ruled out, since the respiratory tract is considered the usual port of entry for these organisms although the presence of the central line in our first case favours its use as the main site for Aspergillus entry and hence it was removed rapidly. In our second case, no venous access device had been in place during the onset of fever, and preceding bacterial sepsis with pneumonia might have caused pulmonary tissue damage, facilitating aspergillus entry along with the use of broad spectrum antibiotics. According to the international literature the strongest risk factor for disseminated aspergillosis is prolonged granulocytopenia in the context of immunosuppressive chemotherapy. In our patients aspergillus endocarditis presented in complete remission from leukemia, and the neutrophil count was normal at the onset of infection. ${ }^{12}$

In conclusion these two cases of Aspergillus endocarditis showed the diversity of presentation of a critical fungal infection in immunocompromised non neutropenic paediatric patients. The prompt diagnosis and initiation of treatment is crucial for a favourable outcome along with the use of double antifungal treatment with liposomal amphotericin and voriconaz which could be later switched to oral voriconazole with a good tissue penetration.

\section{References}

1. Bouza E, Guinea J, Pelaez T, et al. Workload due to Aspergillus fumigatus and the significance of the organism in the microbiology laboratory of a general hospital. J Clin Microbiol 2005;43:2075-9.

2. Rubinstein E, Noriega ER, Simberkoff MS, et al. Fungal endocarditis: analysis of 24 cases and review of the literatureMedicine (Baltimore). 1975;54:331-4.

3. Pierrotti LC, Baddour LM. Fungal Endocarditis, 1995-2000. Chest 2002;122: 302-10.

4. Babatasi G, Massetti M, Chapelier A, et al. Surgical treatment of pulmonary aspergilloma: current outcome. J Thorac Cardiovasc Surg 2000;119:906-12.

5. Chim CS, Ho PL, Yuen ST, Yuen KY. Fungal endocarditis in bone marrow transplantation: case report and review of literature. $\mathrm{J}$ Infect 1998;37:287-91.

6. Petrikkos GL, Skiada A, Samonis G, et al. Native valve Aspergillus endocarditis in two patients with aplastic anaemia. Scand J Infect Dis 2006;38:916-20.

7. Bow EJ, Laverdiere M, Lussier N, et al. Antifungal prophylaxis for severely neutropenic chemotherapy recipients: a meta analysis of randomized-controlled clinical trials. Cancer 2002;94:3230-46.

8. Pemán J, Ortiz R, Osseyran F, et al. Native valve Aspergillus fumigatus endocarditis with blood culture positive and negative for galactomannan antigen. Case report and literature review. Rev Iberoam Micol 2007;24:157-60.

9. Reis LJ, Barton TD, Pochettino A, et al. Successful treatment of Aspergillus prosthetic valve endocarditis with oral voriconazole. Clin Infect Dis 2005;41:7523.

10. Schett G, Casati B, Willinger B, et al. Endocarditis and Aortal Embolization Caused by Aspergillus terreus in a Patient with Acute Lymphoblastic Leukemia in Remission: Diagnosis by Peripheral-Blood Culture Clin Microbiol. 1998;36:3347-51.

11. Berger LA. Imaging in the diagnosis of infections in immunocompromised patients. Curr Opin Infect Dis 1998;11431-6.

12. Millar BC, Jugo J, Moore JE. Fungal endocarditis in neonates and children. Pediatr Cardiol 2005;26:517-36. 